\title{
TRANSPLANTE DE FÍGADO EM HOSPITAL UNIVERSITÁRIO DO RECIFE: RESULTADOS INICIAIS
}

\section{LIVER TRANSPLANTATION IN AN UNIVERSITY HOSPITAL IN RECIFE: INITIAL RESULTS}

\author{
Cláudio Moura Lacerda, TCBC-PE ${ }^{1}$ \\ Paulo Sérgio Vieira de Melo \\ Olival Lucena, ACBC-PE ${ }^{2}$ \\ Américo Amorim ACBC - PE $^{3}$ \\ Herbert Motta Almeida ${ }^{4}$ \\ Leila Beltrão Pereira ${ }^{5}$
}

\begin{abstract}
RESUMO: Objetivo: Apresentar a experiência e enfatizar o aprendizado obtido em um programa incipiente de transplante hepático desenvolvido em Hospital Universitário de Recife-PE. Método: Foram estudados os primeiros 20 pacientes submetidos a transplante ortotópico de fígado, de maneira simplificada, no período de agosto de 1999 a março de 2002. Foram analisadas as indicações, a reserva funcional hepática préoperatória, o volume de sangue transfundido, o tempo de isquemia, o tempo de permanência em UTI, a morbidade e a mortalidade. A cirrose por vírus C foi a indicação de transplante em 9 pacientes (45\%), seguida de cirrose alcoólica em 7 (35\%). Quarenta e cinco por cento dos pacientes foram classificados como CHILD-PUGH A, 35\% como B, e apenas $20 \%$ como C. Resultados: O tempo de isquemia médio foi de $9 \mathrm{~h}$ 09' ( \pm 2 h 33'). Foram utilizadas em média 2,88 $( \pm 2,11)$ unidades de hemácias. A técnica empregada foi a convencional sem bypass em $90 \%$ dos casos e piggyback nos restantes. No pós-operatório, houve um caso de trombose de artéria hepática e outro de veia porta. Oito pacientes apresentaram complicações biliares, todas resolvidas por via endoscópica ou percutânea. A sobrevida global é de 100\%, no período de seguimento de 2 a 32 meses. Conclusão: Conclui-se que é possível realizar transplantes de fígado, com bons resultados, em hospital universitário do Nordeste do Brasil, desde que se reúnam as mínimas condições de estrutura física e recursos humanos.
\end{abstract}

Descritores: Transplante hepático

\section{INTRODUÇÃO}

A Organização Mundial de Saúde considera que 20 pessoas por milhão de habitantes precisam ser submetidas a transplante de fígado a cada ano, numa determinada região. O Nordeste brasileiro tem uma população algo superior a 45 milhões de indivíduos. Assim, estima-se que mais de 900 deveriam ser submetidos ao transplante hepático anualmente. Ocorre que, até 1999, restava a esses

1 Professor Titular de Cirurgia Abdominal da Universidade de Pernanbuco e Chefe do Serviço de Cirurgia Geral do Hospital Universitário Oswaldo Cruz -UPE.

2 Cirurgião do Serviço de Cirurgia Geral do Hospital Universitário Oswaldo Cruz -UPE.

3 Professor Assistente de Cirurgia da Universidade de Pernanbuco.

4 Residente do $3^{\circ}$ ano em Cirurgia Gastroenterológica do Hospital Universitário Oswaldo Cruz -UPE.

5 Professora Titular de Gastroenterologia da Universidade de Pernambuco.

\section{Recebido em 03/05/2002}

Aceito para publicação em 08/10/2002

Trabalho realizado no Serviço de Cirurgia Geral e Transplante Hepático do Hospital Universitário Oswaldo Cruz da Universidade de Pernambuco. 
hepatopatas graves, pobres na sua maioria, a possibilidade de se deslocar para o Sudeste, enfrentar longas filas de espera, na qual a mortalidade é superior a $50 \%$, na esperança de ser contemplado com um novo fígado. Enquanto isso, paradoxalmente, os doadores nordestinos eram desperdiçados, por falta de centro transplantador ${ }^{1}$.

Embora o primeiro transplante hepático no Recife tenha sido realizado em $1993^{2}$, só a partir de agosto de 1999 foi possível retomar e consolidar o programa, que hoje tem entre os já transplantados e na lista de espera, pacientes de praticamente todos os estados do Norte e Nordeste ${ }^{3}$. Visando estimular a criação de centros transplantadores em regiões desatendidas do Brasil, os autores apresentam os primeiros 20 transplantes de fígado realizados no Hospital Universitário Oswaldo Cruz da Universidade de Pernambuco.

\section{MÉTODO}

No período compreendido entre agosto de 1999 e março de 2002, vinte pacientes foram submetidos a transplante hepático com doador cadáver, fígado inteiro. A idade variou de 4 a 65 anos $(45,3 \pm 14,6)$. A indicação mais freqüente do transplante hepático foi a cirrose pelo vírus $\mathrm{C}$, seguida de cirrose alcoólica (Tabela-1). A reserva funcional hepática pré-operatória, segundo os critérios de CHILD-PUGH, são apresentados na Tabela-2. Os pacientes classificados como CHILD-PUGH A eram os portadores de doença colestática ou neoplásica, exceto um caso de cirrose alcoólica com episódios graves de hemorragia digestiva clinicamente intratável. Durante a intervenção, não se realizou

Tabela 1 - Indicações para o transplante hepático $(n=20)$.

\begin{tabular}{lcc}
\hline Diagnóstico & n & \% \\
\hline Cirrose VHC & 9 & 45 \\
Cirrose alcoólica & 4 & 20 \\
Cirrose biliar secundária & 2 & 10 \\
Colangite esclerosante & 2 & 10 \\
Carcinoma fibrolamelar & 1 & 5 \\
Cirrose biliar primária & 1 & 5 \\
Cirrose auto-imune & 1 & 5 \\
Total & 20 & 100 \\
\hline
\end{tabular}

VHC-Virus da hepatite C.
Tabela 2 - Distribuição dos pacientes em relação à reserva funcional hepática pré-operatória, de acordo com os critérios de CHILD-PUGH.

\begin{tabular}{ccc}
\hline CHILD-PUGH & $\mathbf{n}$ & \% \\
\hline A & 9 & 45 \\
B & 7 & 35 \\
C & 4 & 20 \\
Total & 20 & 100 \\
\hline
\end{tabular}

monitorização com cateter de Swan-Ganz em qualquer caso, nem se utilizou tromboelastógrafo na monitorização da coagulação.

Os doadores, captados em Recife (15), Salvador (2), Fortaleza (1), Teresina (1), e Caruaru (1), eram de excelente ou boa qualidade. Empregou-se técnica convencional sem by-pass em 18 casos (90\%) e piggyback em dois (10\%).

Administrou-se aprotinina, na dose de 500.000 unidades a cada duas horas, no transoperatório dos pacientes com sinais pré-operatórios de coagulopatia severa ou moderada.

A reconstrução biliar foi realizada através de colédoco-colédoco anastomose em 18 (90\%) e bilio-digestiva em dois (10\%) dos pacientes. Dreno biliar foi utilizado em três casos, não se empregando drenagem cavitária. Em um paciente, que apresentava hipoplasia acentuada de veia porta ( $2 \mathrm{~mm}$ de diâmetro), impossibilitando uma anastomose adequada, a revascularização portal foi realizada através de anastomose entre a veia gástrica esquerda do receptor $(8 \mathrm{~mm})$ e a porta do doador $(8 \mathrm{~mm})^{4}$.

O esquema de imunossupressão empregado foi à base de corticóides e tacrolimus (FK-506). Os pacientes com vírus $\mathrm{C}$ receberam, adicionalmente, micofenolato mofetil, retirando-se o corticóide em torno do $30^{\circ}$ dia do pós-operatório. A dose de indução do tacrolimus foi de $0,1 \mathrm{mg} / \mathrm{Kg} / \mathrm{dia}$, procurando-se manter o nível sérico entre 15 e $20 \mathrm{ng} / \mathrm{ml}$ no $1^{\circ}$ mês, abaixando-se para 5 a $10 \mathrm{ng} / \mathrm{ml}$ após o $3^{\circ}$ mês.

\section{RESULTADOS}

O tempo cirúrgico, o tempo de isquemia e o número de unidades de concentrado de hemácias administradas durante a operação são apresentados na Tabela-3. O tempo de internação 
Tabela 3 - Tempo cirúrgico, tempo de isquemia e unidades de concentrado de hemácias utilizadas durante a cirurgia.

\begin{tabular}{lcccc}
\hline & Vmín. & Vmáx. & Média & DP \\
\hline Tempo cirúrgico & $7 \mathrm{~h} 10^{\prime}$ & $15 \mathrm{~h} 24^{\prime}$ & $10 \mathrm{~h} 47^{\prime}$ & 2h 23' \\
Tempo de isquemia total & $6 \mathrm{~h}$ & $13 \mathrm{~h} \mathrm{30}$ & $9 \mathrm{~h} \mathrm{09}$ & $2 \mathrm{~h} 33^{\prime}$ \\
Concentrado de hemácias* & 0 & 7 & 2,88 & 2,11 \\
\hline
\end{tabular}

* Unidades administradas; Vmín - Valor mínimo; V máx - Valor máximo; DP - Desvio padrão.

em UTI variou de três a 42 dias, com média de 5,75 dias $( \pm 8,57)$.

Um paciente apresentou trombose tardia de veia porta, evoluindo com ascite. Quinze meses após o transplante, houve revascularização hepatopetal espontânea e desaparecimento da ascite. Uma paciente apresentou trombose da artéria hepática em torno do $30^{\circ}$ dia de pósoperatório, evoluindo com abscesso hepático, drenado por via percutânea. Vinte meses após o transplante, apresenta episódios esporádicos de colangite segmentar, podendo tornar-se candidata a re-transplante ou a hepatectomia.

Houve oito complicações biliares (40\%): disfunção do esfíncter de Oddi em cinco casos, litíase biliar em dois casos e estenose de anastomose biliodigestiva em um paciente. Em todos os casos foram tratados por via endoscópica (papilotomia e litotomia) ou percutânea.

Houve oito episódios de rejeição celular aguda em sete pacientes (35\%), confirmados por biópsia. $\mathrm{O}$ tratamento consistiu de três pulsos de $1 \mathrm{~g}$ de metil-prednisolona em dias consecutivos, seguidos de reciclagem. Houve resposta adequada em todos os casos (Tabela-4).

Não houve mortalidade operatória. A sobrevida atual, no período de seguimento de 2 a 32 meses, é de $100 \%$.

\section{DISCUSSÃO}

O impacto de um programa de transplante hepático numa região carente do ponto de vista da assistência à saúde, vai muito além do benefício prestado aos receptores. Por ser atividade multidisciplinar, de alta complexidade, incompatível com largas margens de erro, incorpora tecnologia e exige constante aprimoramento dos recursos humanos envolvidos direta ou indiretamente. Disso resulta elevação do nível da medicina praticada, inclusive em outras áreas, no hospital e na região, em benefício de todos, justificando o alto custo financeiro do programa. O trabalho de implantação e consolidação de um programa como esse exigiu adequação à realidade de um hospital com estrutura física subótima. Sem abrir mão da agilidade e da utilização de recursos tecnológicos indispensáveis, as condutas foram norteadas pela simplificação e pelo pragmatismo. Assim foi a monitorização transoperatória, a técnica cirúrgica e a condução pósoperatória.

Na presente série não se empregou monitorização com cateter de artéria pulmonar e de tromboelastógrafo. Em nenhum momento esses recursos se mostraram indispensáveis. A complicação hemorrágica mais temível e freqüente no transoperatório do transplante hepático é a fibrinólise. Por

Tabela 4 - Distribuição das complicações pós-operatórias.

\begin{tabular}{|c|c|c|c|}
\hline Complicações & n $(\%)$ & Manuseio & Evolução \\
\hline Rejeição Celular Aguda & $7(35)$ & Metil-Prednisolona & Resolução \\
\hline Complicações biliares & $8(40)$ & Endoscópico / percutâneo & Resolução \\
\hline Trombose de veia porta & $1 \quad(5)$ & Conservador & Resolução \\
\hline Trombose de AH com APF & $1 \quad(5)$ & Drenagem percutânea & Colangite isquêmica \\
\hline
\end{tabular}

AH - Artéria hepática / APF - Abscesso piogênico do fígado. 
Tabela 5 - Vantagens e desvantagens teóricas das três técnicas utilizadas no transplante ortotópico de fígado. Parâmetros avaliados: instabilidade hemodinâmica (IH); dificuldade técnica (DT); tempo de isquemia quente (TIQ); risco de tromboembolismo (RT); estenose cava supra (EC-S); e custo econômico (CE).

\begin{tabular}{lcccccc}
\hline \multicolumn{1}{c}{ Técnica } & I H & DT & TIQ & RT & EC-S & C E \\
\hline Convencional com Bypass & + & + & ++ & ++ & + & ++ \\
Piggyback & ++ & +++ & + & + & ++ & + \\
Convencional sem Bypass & +++ & ++ & ++ & + & + & + \\
\hline
\end{tabular}

isso, em pacientes de risco, empregou-se aprotinina de forma profilática, não se registrando sinais de coagulopatia em qualquer caso $^{5,6}$.

Quanto à técnica cirúrgica, na maioria dos serviços prefere-se a piggyback ${ }^{7}$, preservando-se a veia cava retro-hepática ou a convencional com by pass porto-cava-axilar ${ }^{8,9}$. Na presente série, preferiuse a convencional, porém, sem by pass ${ }^{10}$.

Nesses casos, para contornar os efeitos do pinçamento das veias cava e porta realizou-se administração generosa de volume imediatamente antes e durante a fase anepática (controlando-se a PVC) e diuréticos imediatamente antes e logo após a revascularização do enxerto. Desta forma, protegeuse a função renal do pinçamento vascular e a função pulmonar da hipervolemia que ocorre após o desclampeamento. Na Tabela 5, apresenta-se uma avaliação crítica das três técnicas cirúrgicas que podem ser empregadas no transplante hepático com doador cadáver ${ }^{10,11}$.

Apenas doadores de boa qualidade foram utilizados. Mesmo diante da escassa oferta de enxertos, por conta de um sistema de captação pouco eficiente, e da elevada mortalidade na lista de espera, doadores marginais foram evitados pelo receio do "não-funcionamento primário do enxerto", que implicaria em indicação urgente de re-transplante, que, no contexto da mencionada escassez de doadores, levaria a uma situação dramática. Para adquirir credibilidade no meio, os primeiros dois receptores foram selecionados, evitando-se os casos graves. $\mathrm{O}$ primeiro apresentava um carcinoma fibrolamelar recidivado e o segundo, cirrose biliar primária. A partir de então, afrouxaram-se as exigências em relação à condição clínica dos receptores, operandose doentes mais graves. Todavia, nenhum paciente crítico, de UTI, foi transplantado até a presente data. Isso porque, em geral, no nosso meio, esses pacientes evoluem a óbito antes que se consiga um doador.

A incidência de complicações biliares na presente série $(40 \%)$ aproximou-se da registrada em alguns centros do Brasil ${ }^{12}$ e do exterior ${ }^{13,14,15}$ mas foi bem superior às observadas em estudo multi-cêntrico dos EUA (16 e 24,5\%) que incluíu mais de 6000 transplantes de fígado ${ }^{16}$. Entretanto, na presente série, em todos os casos em que a reconstrução havia sido realizada através de colédoco-coledocostomia foi possível a solução por via endoscópica. Nos pacientes com colédocojejunostomia, a abordagem percutânea seguida de litotomia ou dilatação de anastomose e colocação de stent auto-expansível também foi resolutiva, pelo menos a médio prazo.

Conclui-se que é possível realizar transplante de fígado em hospital público de região carente em termos de assistência à saúde, com bons resultados, desde que se reúnam equipe multidisciplinar treinada e mínima estrutura física. 


\begin{abstract}
Background: This paper describes the experience and emphasizes the learning process with a program in liver transplantation developed at a University Hospital in Recife-PE. Methods: Twenty patients were submitted to conventional orthotopic liver transplantation in a simplified way during the period from August 1999 to March 2002. Indications, liver function, blood transfusion, allograft ischemia time, intensive care period and morbidity and mortality rates were analyzed. VHC cirrhosis was the transplant indication in 9 (45\%) patients, followed by alcoholic cirrhosis in 4 (20\%) patients. Fifty per cent of the transplanted patients were classified in CHILD-PUGH as B, $40 \%$ as A, and $10 \%$ as C. Results: The mean allograft ischemia time was 9 hours and 9 minutes ( $\left.\pm 2 \mathrm{~h} 33^{\prime}\right)$. The average of blood transfusion was 2,8 $( \pm 2,11)$ units. The operative technique was conventional without bypass in 18(90\%) cases. There was one case of hepatic artery thrombosis and one of portal vein thrombosis. Eight patients (40\%) presented biliary complications wich were resolved using endoscopic or percutaneus methods. A $100 \%$ of overall survival rate was achieved during a follow up of 2 to 32 months. Conclusion: The results of this initial study show that it is possible to perform liver transplantation with good results at a university hospital in the northeast region when a minimum of physical structure and human resources is assembled.
\end{abstract}

Key words: Liver transplantation.

\section{REFERÊNCIAS}

1. Registro Brasileiro de Transplantes. Ano V - no $4-$ outubro / dezembro, 1999, pg. 15, 23.

2. Lacerda CM, Sette M, Maia M - Carcinoma fibrolamelar recidivado: O primeiro caso de transplante de fígado no Recife-Pe. An Fac Méd Univ Fed Pernamb. Recife, 1994, 39:125-128.

3. Lacerda CM, Melo PSV, Amorim A, et al. - Transplante de Fígado em Hospital Público de Pernambuco. An Fac Méd Univ Fed Pernamb. Recife, 2000, 45 (2).

4. Lacerda CM, Melo PSV, Amorim A - A veia gástrica esquerda como alternativa de revascularização portal no transplante hepático. Acta Cir Bras, Fevereiro 2002, vol. 17 no. 1 .

5. García-Huete L, Domenech P, Sabate A, et al. - The prophylactic effect of aprotinin on intraoperative bleeding in liver transplantation: a randomized clinical study. Comment.Hepatology, 1998, Apr; 27(4):1169-7.

6. Molenaar IQ, Veldman M, Begliomini B, et al. - Improved early graft survival in patients receiving aprotinin during orthotopic liver transplantation. Transplant Proc, 2001 Feb-Mar; 33(1-2):1345-1346.

7. Tzakis A, Todo S, Starzl TE - Piggyback orthotopic liver transplantation with preservation of the inferior vena cava. Ann Surg, 1989, 210: 549-552.

8. Starzl TE - Experience of hepatic transplantation. WB Saunders Co. Philadelphia. 1969.

9. Griffith BP, Shawn Jr BW, Hardesty RL, et al. -Venovenous bypass without systemic anticoagulation for transplantation of the human liver. Surg Gynecol Obstet, 1985, 160:271-272.
10. Lacerda CM, Melo PSV, Amorim A, et al. -Bypass ou piggyback no transplante ortotópico de fígado. Há sempre necessidade? An Fac Med Univ Fed Pernamb. Recife, 2001; 46(1), p.44-48.

11. Hosein M, Osama A, Bagous WA, et al. - Choice of surgical technique influences perioperative outcomes in liver transplantation. Ann Surg, 2000, Jun; 231(6):814-823.

12. Freitas AC, Coelho JC, Parolin M, et al. - Fatores de Risco e Conduta nas Complicações do Trato Biliar no Transplante Hepático. Rev Col Bras Cir, 2000; 27(6): 359-365.

13. Li S, Stratta RJ, Lagnas AN, et al. - Diffuse Biliary tract injury after orthotopic liver transplantation. AM J Surg, 1992, 164:536-540.

14. Greif F, Bronsther Ol, Van Thiel DH, et al. - The incidence, timing and management of Biliary tract complications after orthotopic liver transplantation. Ann Surg, 1994, 219:40-45.

15. Sossenheimer M, Slivka A, Carr-Lcke D -Management of extra-hepatic biliary after orthotopic liver transplantation: review of the literature and results of a multicenter survey. Endoscopy, 1996, 28:565-571.

16. Reknimitr R, Sherman S, Fogel E, et al. - Biliary tract complications after orthotopic liver transplantation with choledocholedochostomy anastomosis: endoscopic fidings and results of therapy. Gastrointestinal Endoscopy, 2002, 55(2):224-231.

Endereço para correspondência:

Cláudio Moura Lacerda

Rua Prof a Anunciada Rocha Melo, 97/501

Recife - PE - CEP: 50710-390

E-mail: cmlacerda1@hotmail.com 